\title{
Auswirkungen einer intensivierten Therapie
}

Fragestellung: Welche Inzidenz und Prädiktoren haben Hypoglykämien bei Typ-2-Diabetikern, deren Behandlung aufgrund einer insuffizienten Blutzuckereinstellung intensiviert wurde?

Hintergrund: Die Hypoglykämie ist eine unerwünschte Nebenwirkung bei der Diabetes-Therapie [1]. Die schwere Hypoglykämie gilt als Prädiktor kardiovaskulärer Ereignisse bzw. der Mortalität [2-4]. In das DiaRegis, ein prospektives deutsches Register, wurden 3810 Typ-2-Diabetiker aufgenommen, die wegen einer unzureichenden Blutzuckerkontrolle mit ein oder zwei oralen Antidiabetika in der Therapie intensiviert wurden.

Patienten und Methodik, Ergebnisse: Von 3347 Patienten mit verfügbaren Daten erlitten 473 (14,1\%) eine Hypoglykämie unterschiedlichen Ausprägungsgrades binnen 12 Monaten nach Therapieintensivierung. 0,4\% der Patienten mit Hypoglykämien wurden stationär aufgenommen (im Mittel 1,3 $\pm 0,6$ Episoden), $0,1 \%$ der Hypoglykämien bedurften medizinischer Hilfe $(1,0 \pm 0,0), 0,8 \%(1,1 \pm 0,5)$ der Patienten benötigten Fremdhilfe und $10,1 \%(3,4 \pm 3,7)$ benötigten keine spezifische Unterstützung (Abb. 1). Die Hypoglykämien (8,0\% inapparent) waren mit einer längeren Diabetesdauer, höheren $\mathrm{HbA}_{1 c}$-Ausgangswerten, einem anamnestischen Nikotinabusus und einer höheren Rate an Begleiterkrankungen wie einer KHK, pAVK, Amputation, Herzinsuffizienz, peripherer

Tschöpe D, Bramlage P, Binz C et al. Incidence and predictors of hypoglycaemia in type 2 diabetes - an analysis of the prospective DiaRegis registry. BMC Endocr Disord. 2012;12(1):23.
Neuropathie, diabetischer Retinopathie und einer klinisch relevanten Depression bei Studienbeginn assoziiert. Weitere Prädiktoren waren eine Hypoglykämie in der Anamnese, Retinopathie, Depression, In-

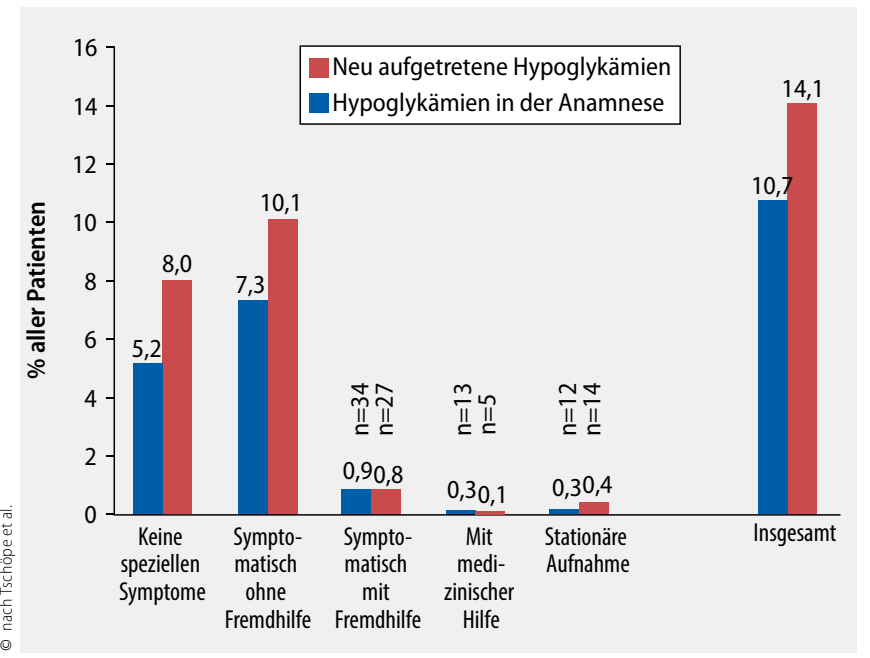

1 Hypoglykämieraten 12 Monate vor und 12 Monate nach Therapieintensivierung. Der Anstieg ist nicht zu leugnen.

sulintherapie und die Blutzuckerselbstmessung. Nicht dazu gehörte die Vor-Therapie mit Sulfonylharnstoffen. Glitazone, DPP-4-Hemmern und GLP-1-Analoga waren mit einem reduzierten Hypoglykämie-Risiko assoziiert.

Schlussfolgerungen: Die Hypoglykämie ist häufig bei ambulanten Patienten nach Therapieintensivierung. Besondere Aufmerksamkeit ist geboten bei Hypoglykämien in der Anamnese, bei mikrovaskulären Erkrankungen und bei Patienten mit Insulintherapie. Glitazone, DPP-4-Inhibitoren oder GLP-1-Analoga sind mit einem signifikant reduzierten Risiko assoziiert.

\section{- Kommentar von Dr. med. Thorsten Siegmund}

\section{Individualisierter Ansatz heißt die Devise}

Versorgungsforschung wie hier anhand eines Diabetesregisters wird nach wie vor noch nicht ausreichend genutzt, um Therapien oder Therapiemaßnahmen im Alltag zu überprüfen. Randomisierte bzw. auch randomisiert doppelblinde Studien sind sicher vielfach wichtig, bilden aber oft nicht die Versorgungsrealität ab. Erfreulich ist, dass sich ein Großteil der Ergebnisse des Registers mit der aktuellen Philosophie in der Diabetologie deckt. Relevant ist ein individualisierter Ansatz bezüglich Therapieziel und Therapiemaßnahmen. Besondere Vorsicht ist geboten bei Patienten mit langer Diabetesdauer und vielen Begleiterkrankungen.

\section{Literatur:}

1. Frier BM. Diabetologia 2009, 52(1):31-34.

2. Dluhy RG, McMahon GT. N Engl J Med 2008, 358(24):2630-33.
3. Cryer PE. Am J Med 2011, 124(11):993-96.

4. Boucai L, Southern WN, Zonszein. J Am J Med 2011,124(11):1028-35.

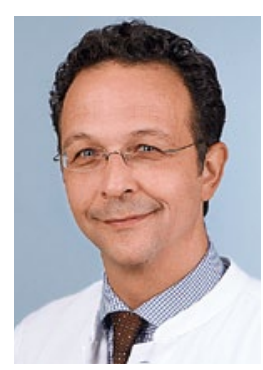

Dr. med. Thorsten Siegmund

Klinik für Endokrinologie, Diabetologie und Angiologie Klinikum München Bogenhausen, Städt. Klinikum München GmbH Englschalkinger Straße 77, 81925 München thorsten.siegmund@klinikum-muenchen.de 